\title{
Dried Blood Spot Sampling with LC-MS Analysis for Routine Therapeutic Caffeine Monitoring in Neonates
}

\author{
Graham Lawson, ${ }^{1}$ Parul Patel, ${ }^{2}$ Hussain Mulla, ${ }^{2}$ and Sangeeta Tanna ${ }^{1}$ \\ ${ }^{1}$ Leicester School of Pharmacy, Faculty of Health and Life Sciences, De Montfort University, The Gateway, Leicester LE1 9BH, UK \\ ${ }^{2}$ Centre for Therapeutic Evaluation of Drugs in Children, University Hospitals of Leicester NHS Trust, Glenfield Hospital, Groby Road, \\ Leicester LE3 9QP, UK
}

Correspondence should be addressed to Sangeeta Tanna, stanna@dmu.ac.uk

Received 27 September 2012; Accepted 15 October 2012

Academic Editors: M. C. Bruzzoniti, L. Chen, and C. M. Remsberg

Copyright (C) 2012 Graham Lawson et al. This is an open access article distributed under the Creative Commons Attribution License, which permits unrestricted use, distribution, and reproduction in any medium, provided the original work is properly cited.

\begin{abstract}
A liquid chromatography-mass spectrometry (LC-MS) method was developed and validated for the determination of therapeutic levels of caffeine in dried blood spot (DBS) samples. Caffeine is used in the treatment of Apnoea of Prematurity (AoP) in newborn children. Calibration DBS samples were prepared by spotting $15 \mu \mathrm{L}$ of whole blood spiked with the analyte onto specimen collection cards. $3 \mathrm{~mm}$ disks cut from the centre of the DBS were extracted in methanol containing the internal standard. The extract was separated using a Zorbax Eclipse Plus C18 column and the MS, operated in electrospray positive ion mode, used single ion monitoring at $\mathrm{m} / \mathrm{z} 195$ for caffeine and $\mathrm{m} / \mathrm{z} 198$ for the IS. The overall extraction recovery of caffeine from spiked blood spots was demonstrated to be $44-47 \%$. Validation of the microanalytical method showed good precision (coefficient of variation) and accuracy (relative error) and specificity and was linear within the tested calibration range 500-25000 ng/mL for caffeine. Investigation of different specimen collection papers revealed different matrix effects with significant ion suppression from the FTA Elute paper itself. Requiring only a microvolume $(15 \mu \mathrm{L})$ blood sample for analysis, the developed DBS based microanalytical method has the potential to facilitate the routine monitoring of caffeine in neonates.
\end{abstract}

\section{Introduction}

The analysis of dried blood spots (DBS) is used routinely for newborn screening where the low volume blood sample is obtained from a simple heel prick procedure [1]. Advances in instrumental techniques [2] have enabled the analysis of DBS samples to be expanded to include therapeutic drug monitoring $[3,4]$, paediatric pharmacokinetic $(\mathrm{PK})$ studies [5-8], and metabolite identification investigations $[9,10]$. In this investigation a DBS analytical approach is developed for the routine monitoring of caffeine, used to treat Apnea of Prematurity (AoP) in neonates, that is, children less than 1 month old. Despite there being many references in the literature to the analysis of caffeine in blood, plasma, urine, and saliva there are only two citing the determination of caffeine in dried blood spots (DBS). The GC analysis reported by Brazier et al. [11] required solvent extraction followed by derivatisation with methyxanthine prior to measurement. More recently a DBS method without the need for several work-up steps and compound derivatisation has been established [12]. This method, based on LC-MS/MS analysis, has a lower limit of quantification (LLOQ) of $250 \mathrm{ng} / \mathrm{mL}$ based on a $15 \mu \mathrm{L}$ DBS sample.

The aim of this investigation was to develop and validate a caffeine DBS method using a simple LC-MS instrument. The International guidelines $[13,14]$ on bioanalytical validation provide guidance on the range of tests to be carried out and the criteria which developed methods must meet to be considered suitable for drug monitoring in patients. A working liquid chromatography-mass spectrometry (LCMS) with selected ion monitoring (SIM) method for the quantification of caffeine was established and subjected to an assessment of accuracy, precision, selectivity, sensitivity, and matrix effect in line with these requirements.

\section{Experimental}

The methodology and validation results from investigations of caffeine DBS quantification by LC-MS are presented in this 
section. All the research carried out was approved by the De Montfort University ethical committee.

2.1. Chemicals and Materials. Caffeine (1,3,7-trimethylxanthine) $\geq 99.0 \%$ (HPLC) and the internal standard (IS) caffeine-trimethyl- ${ }^{13} \mathrm{C}_{3} \quad 1 \mathrm{mg} / \mathrm{mL} 99 \%$ were obtained from Sigma-Aldrich (Poole, UK). FTA Elute paper and Harris punch and cutting mat were purchased from Whatman, UK (Part of GE Healthcare). Blood collection tubes (containing EDTA) and EDTA coated capillary tubes were obtained from Sarstedt (Leicester, UK). HPLC grade water, acetonitrile, and methanol and formic acid $\geq 98 \%$ were obtained from Fisher Scientific (Loughborough, UK). Autosampler vials with $0.3 \mathrm{~mL}$ inserts, eppendorf cups, and volumetric pipettes (Eppendorf Research) were obtained from Fisher Scientific (Loughborough, UK). The vortex (TopMix Wizard X), shaker (IKA VXR Vibrax), and weighing scales (Mettler Toledo XS105) were obtained from Fisher Scientific (Loughborough, UK). Specimen collection filter paper S\&S 903 was obtained from Fisher Scientific (Loughborough, UK). Filter paper Ahlstrom 226 was supplied by ID Biological systems (South Carolina, USA). Sample glass tubes and polythene bags for storage of blood spot specimens were obtained from Richardson's of Leicester (Leicester, UK) and desiccant sachets were supplied by Sud-Chemie (Northwich, UK). Blank blood (EDTA) for method validation and patient sample analysis was obtained from healthy adult volunteers asked to abstain from dietary sources of caffeine for at least two weeks.

2.2. Preparation of Caffeine Stock and Working Solutions. A stock solution of caffeine was prepared by dissolving $0.05 \mathrm{~g}$ in $5 \mathrm{~mL}$ of methanol: water $(80: 20, \mathrm{v} / \mathrm{v})$ to produce a $10 \mathrm{mg} / \mathrm{mL}$ concentration. This was diluted with methanol: water $(50: 50, \mathrm{v} / \mathrm{v})$ to produce a $1000 \mu \mathrm{g} / \mathrm{mL}$ working solution which was then used to make a spiking solution for each calibration level. The final concentrations of spiking solutions were $5.25,10.5,21,105,168,315,420$, and $525 \mu \mathrm{g} / \mathrm{mL}$.

\subsection{Preparation of Calibration and Calibration of Dried Blood} Spots. Blood samples with known concentrations of caffeine were prepared by diluting $25 \mu \mathrm{L}$ of each spiking solution with $500 \mu \mathrm{L}$ of blank human whole blood. The final concentration of the blood calibration standards were 250, 500, 1000, 5000, 8000, 15000, 20000, and $25000 \mathrm{ng} / \mathrm{mL}$.

2.4. Extraction of Dried Blood Spots. Calibration and validation blood spot standards were prepared by pipetting $15 \mu \mathrm{L}$ volumes of spiked blood onto FTA Elute paper. These were left to dry at room temperature overnight and were then stored at room temperature in sealed plastic bags with desiccant.

The dried blood spot extraction solution was prepared as above to provide a $1000 \mu \mathrm{g} / \mathrm{mL}$ concentration of the caffeinetrimethyl ${ }^{13} \mathrm{C}_{3}$ (IS) which was then diluted with methanol to produce a final concentration of $500 \mathrm{ng} / \mathrm{mL}$.
The extraction procedure used to recover caffeine was as follows.

(i) A $3 \mathrm{~mm}$ disc was punched out from the centre of DBS samples and transferred to a tube to which $100 \mu \mathrm{L}$ of methanol containing $500 \mathrm{ng} / \mathrm{mL}$ of IS was added.

(ii) The tubes contents were mixed using a vortex and extraction was facilitated by gentle shaking for 60 minutes.

(iii) Extracts were transferred to a clean tube and vortexed briefly prior to LC-MS analysis.

2.5. LC-MS Sample Analysis. Samples were analysed using an Agilent 1200 LCMS system operated in the positive ion electrospray mode with selected ion monitoring (SIM) of the $[\mathrm{M}+\mathrm{H}]^{+}$peaks of caffeine and the internal standard.

The chromatographic system consisted of an Agilent 1100 series quaternary solvent delivery pump, autosampler, and vacuum degasser. Caffeine was analysed on a Zorbax Eclipse Plus C18 column $(150 \mathrm{~mm} \times 2.1 \mathrm{~mm}$ i.d., $3.5 \mu \mathrm{m})$ with a C18 guard column (Phenomenex, Macclesfield UK, $3.0 \times 4.0 \mathrm{~mm}$ ) under isocratic conditions. Mobile phases A (water with $0.13 \%$ formic acid) and B (100\% acetonitrile) were held at $18 \%$ and $82 \%$, respectively, over a run time of 5 minutes. The column oven temperature was set to $40^{\circ} \mathrm{C}$ and the flow rate was $200 \mu \mathrm{L} / \mathrm{min}$. The injection volume was $10 \mu \mathrm{L}$.

MS detection was performed with an Agilent 1200 mass spectrometer with a positive ESI source. The system was calibrated on a daily basis using a tuning mixture supplied by Agilent Technologies. The ionisation source settings optimised to give maximum caffeine signal intensity were as follows: fragmentor voltage, $95 \mathrm{~V}$; drying temperature, $300^{\circ} \mathrm{C}$; capillary voltage, $3000 \mathrm{~V}$; nebuliser pressure, 25 psig; nitrogen gas flow, $7 \mathrm{~L} / \mathrm{min}$. The protonated molecular ion $[\mathrm{M}+\mathrm{H}]^{+}$of caffeine at $\mathrm{m} / z 195$ and IS at $\mathrm{m} / z 198$ was monitored using SIM mode. A dwell time of $289 \mathrm{msec}$ was used for each ion. The software programme Chemstation (series B.1.3, Agilent Technologies) was used to operate the system and acquire all data. Microsoft Excel 2007 (Microsoft Corporation, USA) and Chemstation were used to analyse the data.

\subsection{Validation Procedures}

2.6.1. Selectivity. To demonstrate the selectivity of the LCMS method, blank blood spots and caffeine spiked blood spots were analysed and subsequently processed. Representative extracted ion chromatograms from the LC-MS data at $m / z 195$ and $m / z 198$ were generated to show that other components that could be present in the sample matrix or from the sampling paper were not interfering with the signal from the target analyte.

2.6.2. Linearity and Sensitivity. Caffeine calibration standards were prepared in replicate $(n=5)$ and analysed on three separate days. A calibration plot of analyte/IS peak area ratio against nominal caffeine concentration was produced 


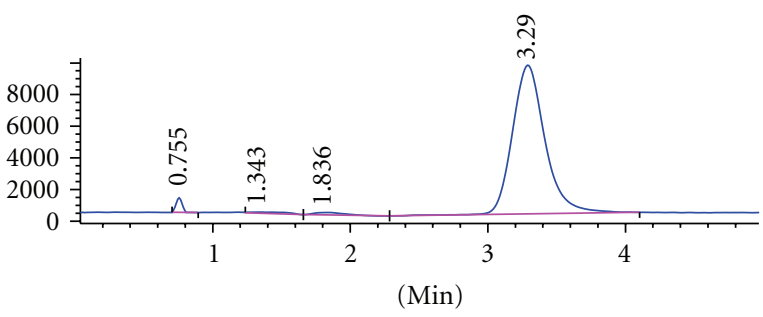

(a) TIC

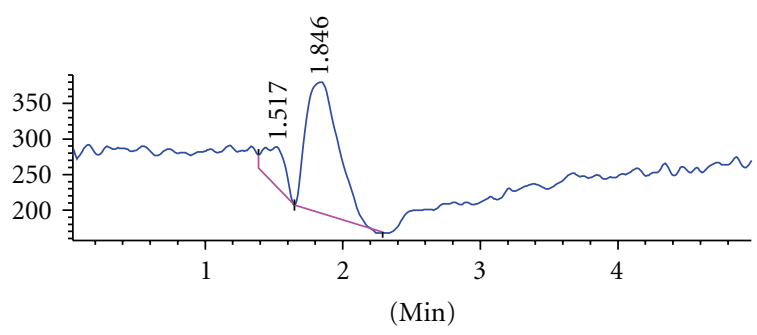

(b) EIC $m / z 195$

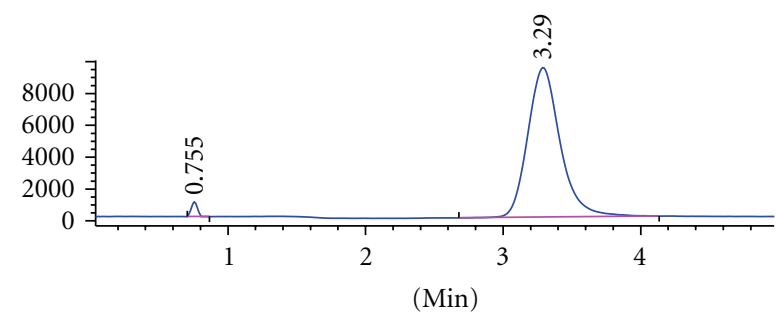

(c) $\mathrm{EIC} m / z 198$

FIGURE 1: A representative LC-MS chromatogram of a blank DBS sample extracted with IS where (a) is the total ion chromatogram (TIC), (b) is the extracted ion chromatogram (EIC) for $m / z$ 195, and (c) is the EIC for $m / z 198$.

and an equally weighted linear regression was applied. The quantification limits of caffeine in the DBS extracts were determined. The limit of quantification was based on a signal-to-noise ratio of 10 in a $0.5 \mathrm{~min}$ window around the elution time of the analyte in an analytical blank.

2.6.3. Accuracy and Precision. The assay accuracy and precision was assessed by analysing a calibration with 7 concentration levels $(n=5)$ in triplicate on three separate days.

2.6.4. Matrix Effects. The matrix effect is a general term used to describe factors which affect the ionization efficiency of a compound in an electrospray source following the extraction of a biological fluid with an organic solvent. Phospholipids have been identified as an important endogenous source of matrix effects and are present in different amounts in various biological fluids including blood, plasma, and urine $[15,16]$.

The effect of the matrix on the resulting caffeine LC-MS signal intensity was investigated at concentrations of 500 and $25000 \mathrm{ng} / \mathrm{mL}$ in replicate $(n=5)$. An assessment was made by comparing the peak area of caffeine spiked into blank DBS extracts with that of caffeine in solvent. A $90 \mu \mathrm{L}$ volume of blank DBS extract or $100 \%$ pure methanol was added to $10 \mu \mathrm{L}$ of caffeine spiking solution. The matrix effect was calculated using peak area by the following equation:

$$
\text { Matrix effect } \%=\frac{\text { Response in DBS extract }}{\text { Response in pure solvent }} \times 100 \text {. }
$$

2.6.5. Recovery of Caffeine from Dried Blood Spots. The overall efficiency of the extraction method utilising $100 \%$ methanol was investigated at DBS concentrations of 500, 8000 , and $25000 \mathrm{ng} / \mathrm{mL}$. At each concentration level replicate ( $n=5) 15 \mu \mathrm{L}$ spots were made and allowed to dry. The entire DBS was subsequently punched out and extracted with
$100 \%$ methanol. Recovery was determined by comparing the peak area obtained with DBS extracts to those obtained from caffeine in solvent containing an amount which assumes $100 \%$ recovery. Recoveries were calculated from the following equation:

$$
\text { Recovery } \%=\frac{\text { Response in DBS extract }}{\text { Response in pure solvent }} \times 100 \text {. }
$$

2.6.6. Stability Considerations. The room temperature stability of caffeine solutions was investigated in replicate $(n=5)$ using the $500 \mathrm{ng} / \mathrm{mL}$ caffeine spiking solution. The peak area obtained from a freshly prepared solution was compared to that from a solution which had been stored for 48 hours.

Caffeine stability in DBS samples was determined by comparing the data from spots containing $1000 \mathrm{ng} / \mathrm{mL}$ and $20000 \mathrm{ng} / \mathrm{mL}$, after 6 days storage at room temperature, with the data obtained from freshly prepared DBS samples. The spots were stored in a sealed plastic bag with a desiccant.

\section{Results and Discussion}

3.1. Selectivity. Under the chromatographic conditions a run time of 5 minutes was achieved with coelution of caffeine and IS at a retention time of 3.3 minutes. Method selectivity was demonstrated following the analysis of DBS samples collected from two individual human subjects. There were no significant ( $\geq 20 \%$ of LLOQ peak area) interferences observed in any of these chromatograms at the retention time of analyte and IS. The selectivity of the method is shown in the representative chromatograms, Figures 1 and 2. The LC-MS trace (Figure 1) obtained from the extract of a blank DBS sample shows the absence of any interfering peak at the retention time for caffeine. The baseline lift off is a result of the very low signal level recorded. The data obtained for 


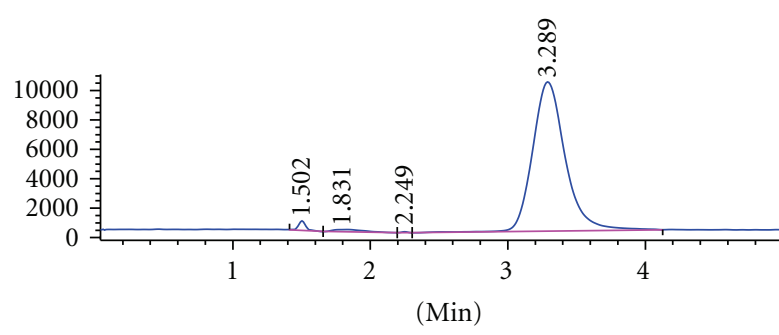

(a) TIC

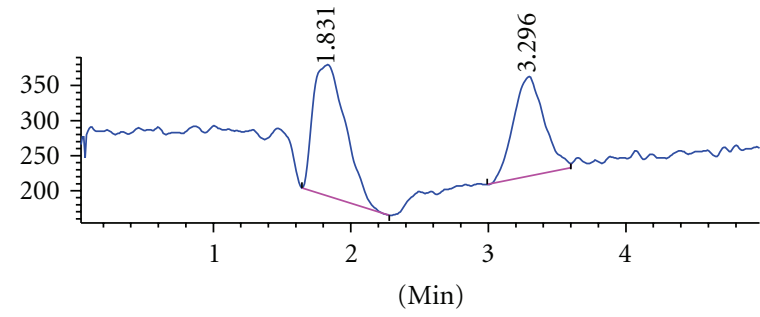

(b) EIC $m / z 195$

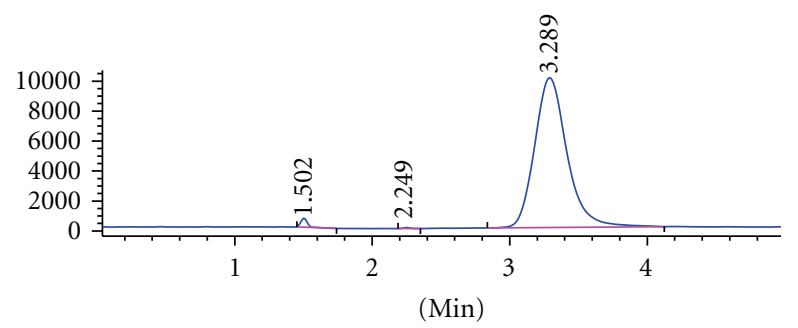

(c) $\mathrm{EIC} m / z 198$

FIGURE 2: A representative LC-MS chromatogram of a spiked DBS at $500 \mathrm{ng} / \mathrm{mL}$ extracted with IS where (a) is the TIC, (b) is the EIC for $m / z$ 195 , and (c) is the EIC for $m / z 198$.

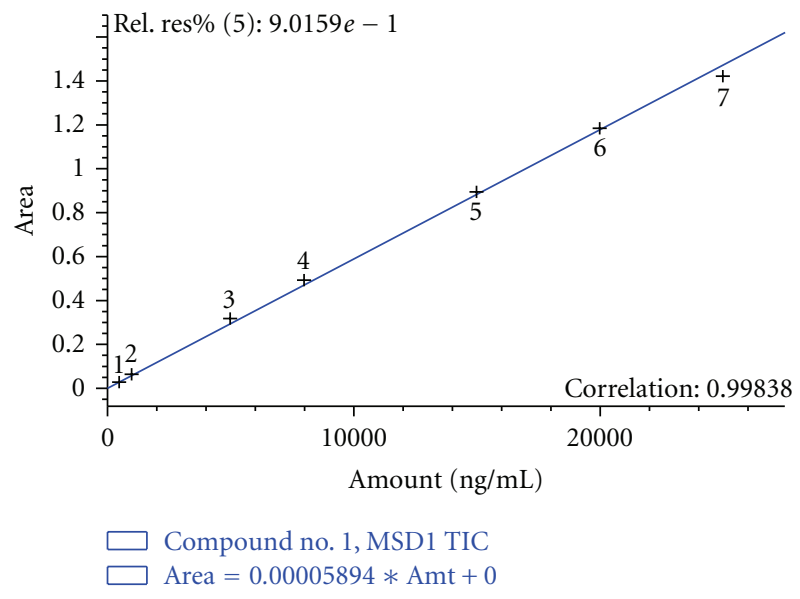

FIGURE 3: Calibration plot of caffeine concentration against mean $(n=5)$ analyte to IS peak area.

a spiked $500 \mathrm{ng} / \mathrm{mL}$ sample are shown in Figure 2. Signal-tonoise ratios were calculated by dividing peak height by the baseline noise (where noise is given as six times the standard deviation of the linear regression).

3.2. Linearity and Sensitivity. A calibration plot of caffeine concentration against analyte/IS peak area ratio showed that the response was linear $\left(r^{2} \geq 0.99\right)$ over the calibration concentration range of 500 to $25000 \mathrm{ng} / \mathrm{mL}$ (Figure 3). The lower limit of quantification LLOQ with a signal-to-noise ratio of $\geq 10$ and $C V \%$ and $\mathrm{RE} \%$ less than $20 \%$ was determined to be $500 \mathrm{ng} / \mathrm{mL}$.

3.3. Accuracy and Precision. The assay accuracy and precision was assessed by analysing a calibration with 7 concentration levels $(n=5)$ in triplicate on three separate days. Intraday CV\% and RE\% was within the acceptable 20\% limit at the LLOQ and within $15 \%$ for all other concentrations. In addition, the interday CV\% and RE\% was within $15 \%$ (Table 1).

3.4. Matrix Effect. Initial results indicated substantial ion suppression $(\geq 15 \%)$ at both concentrations. Clark et al. [17]. showed that FTA Elute papers produced the most marked matrix effects and therefore comparisons were made using S\&S 903 filter paper. Extracts of both papers using $100 \%$ methanol were spiked with caffeine and were then compared with similar caffeine concentration solutions in $100 \%$ methanol. Figure 4 shows the extent of the matrix effect observed under each of the experimental conditions. Components within extracts of blank DBS samples on FTA Elute paper resulted in 32 and 40\% ionisation suppression of the caffeine signal under ESI at 500 and $25000 \mathrm{ng} / \mathrm{mL}$, respectively. Results from the FTA Elute paper showed a similar matrix effect on the caffeine signal suggesting that chemical components within FTA Elute paper rather than endogenous components in blood are responsible for the ion suppression. Under identical chromatographic conditions using paper S\&S 903 no significant matrix effects were observed. This provided further evidence of matrix effects arising from the impregnated chemicals in FTA Elute paper. These results are in agreement with the findings of a study of three different paper types (FTA Elute, FTA, and Ahlstrom 226) where despite the severity of the matrix effect observed for some compounds, FTA Elute was found to be the only paper that enablled an acceptable degree of accuracy and precision for greater than $80 \%$ of test compounds [17]. On that basis the authors concluded that FTA Elute was the preferred choice of paper substrate for method development. 
TABLE 1: Intraday and interday accuracy (RE\%) and precision (CV\%) results for the LC-MS caffeine DBS method $n=5$.

\begin{tabular}{|c|c|c|c|c|c|c|c|c|}
\hline \multirow{2}{*}{\multicolumn{2}{|c|}{ Nominal conc. $(\mathrm{ng} / \mathrm{mL})$}} & \multicolumn{7}{|c|}{ Intraday } \\
\hline & & 500 & 1000 & 5000 & 8000 & 15000 & 20000 & 25000 \\
\hline \multirow{4}{*}{ run 1} & Mean conc. $(\mathrm{ng} / \mathrm{mL})$ & 441.8 & 1034.2 & 5346.7 & 8325.0 & 15138.9 & 20061.8 & 24087.7 \\
\hline & SD & 75.0 & 20.2 & 698.2 & 339.6 & 668.4 & 1668.9 & 140.1 \\
\hline & $\mathrm{RE} \%$ & -11.8 & 3.2 & 6.8 & 3.9 & 0.8 & 0.1 & -3.8 \\
\hline & $\mathrm{CV} \%$ & 17.0 & 2.0 & 13.1 & 4.1 & 4.4 & 8.3 & 4.7 \\
\hline \multirow{4}{*}{ run 2} & Mean conc. $(\mathrm{ng} / \mathrm{mL})$ & 562.0 & 1113.4 & 5237.2 & 7820.4 & 15204.0 & 17404.8 & 21902.6 \\
\hline & $\mathrm{SD}$ & 38.3 & 92.9 & 182.4 & 202.2 & 2112.4 & 604.3 & 1519.3 \\
\hline & $\mathrm{RE} \%$ & 12.4 & 11.3 & 4.7 & -2.2 & 1.4 & -13.0 & -12.4 \\
\hline & $\mathrm{CV} \%$ & 6.8 & 8.3 & 3.5 & 2.6 & 13.9 & 3.5 & 6.9 \\
\hline \multirow{9}{*}{ run 3} & Mean conc. $(\mathrm{ng} / \mathrm{mL})$ & 503.6 & 971.5 & 4970.3 & 7387.8 & 15702.9 & 21355.2 & 24908.2 \\
\hline & SD & 60.1 & 38.9 & 341.1 & 318.0 & 1466.4 & 1766.7 & 2160.4 \\
\hline & $\mathrm{RE} \%$ & 0.7 & -2.8 & -0.6 & -7.7 & 4.7 & 6.8 & -0.4 \\
\hline & $\mathrm{CV} \%$ & 12.4 & 4.0 & 6.9 & 4.3 & 9.3 & 8.3 & 8.7 \\
\hline & & \multicolumn{7}{|c|}{ Interday } \\
\hline & Mean conc. $(\mathrm{ng} / \mathrm{mL})$ & 502.4 & 1039.7 & 5184.7 & 7844.4 & 15348.6 & 19607.3 & 23632.8 \\
\hline & $\mathrm{SD}$ & 75.1 & 77.6 & 456.9 & 469.6 & 1485.7 & 2165.5 & 2070.2 \\
\hline & Average RE\% & 0.4 & 3.9 & 3.6 & -2.0 & 2.3 & -2.0 & -5.5 \\
\hline & Overall CV\% & 14.9 & 7.5 & 8.8 & 6.0 & 9.7 & 11.0 & 8.8 \\
\hline
\end{tabular}

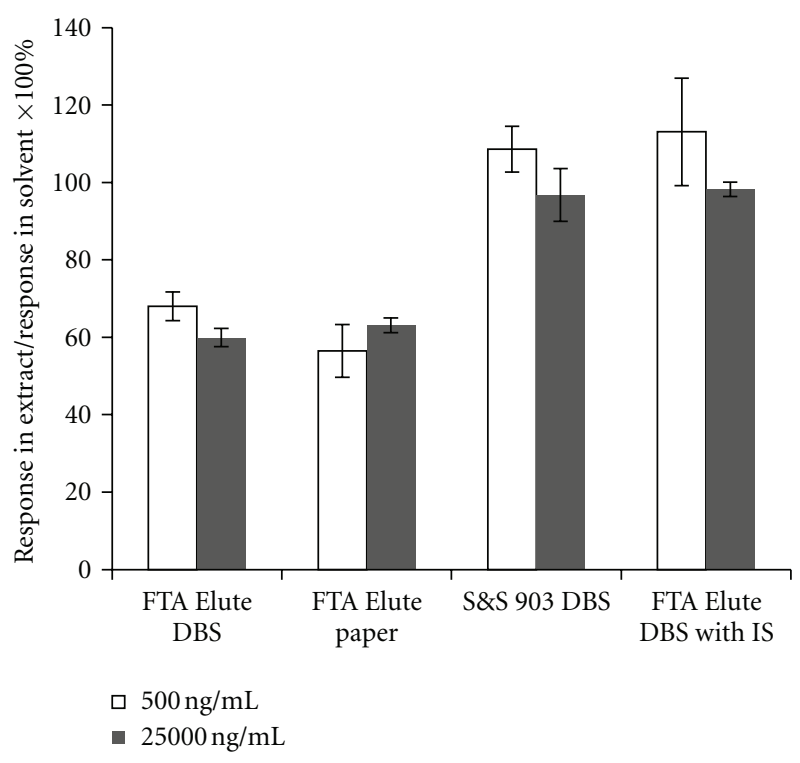

FIGURE 4: Matrix effect observed for caffeine at two concentrations in FTA Elute DBS extract, FTA Elute paper extract, 903 paper DBS extract, and FTA Elute DBS extract containing IS. Results are presented as a percentage of the peak area observed in pure solvent $n=5$.

Several approaches have been used to remove matrix effects including improved sample clean-up [18] or the use of a labeled IS to compensate for the matrix effect [19]. The inclusion of the IS [19] was used to compensate for this problem and the matrix effect \% was calculated using the analyte/IS peak area ratio. After the inclusion of IS the observed matrix effect was within the accepted limit of $15 \%$ at both concentrations.
TABLE 2: Recovery of caffeine from DBS at three concentrations $n=$ 5 .

\begin{tabular}{lccc}
\hline & \multicolumn{3}{c}{ Nominal conc. (ng/mL) } \\
& 500 & 8000 & 25000 \\
\hline Recovery (\%) & 47.0 & 44.2 & 44.9 \\
SD & 7.4 & 2.3 & 3.0 \\
Precision (CV\%) & 15.6 & 5.2 & 6.6 \\
\hline
\end{tabular}

3.5. Recovery of Caffeine from DBS Samples. The overall efficiency of the extraction method utilising 100\% methanol was investigated at DBS concentrations of 500, 8000, and $25000 \mathrm{ng} / \mathrm{mL}$. The overall recovery was relatively low (44 to $47 \%$ ) but consistent and reproducible across the calibration range of the assay (Table 2).

3.6. Stability. The variation in the peak areas from the caffeine stock solutions was found to be $2.8 \%$ (CV, 1.3\%). Indicating stability of the solutions stored at room temperature for 48 hours.

Caffeine was shown (Table 3 ) to be stable on FTA elute paper with the differences in data between stored and fresh samples being less than $15 \%$.

3.7. Carryover. Sample carryover was assessed by analysing blank DBS extracts following the analysis of the highest DBS concentration $(25000 \mathrm{ng} / \mathrm{mL})$ in the calibration range. No significant peak ( $>20 \%$ of LLOQ peak area) was observed in blank DBS chromatograms. Similarly, potential contamination resulting from the punch was assessed by analysing two blank DBS discs cut from the same punching device previously used to cut out 10 discs of blood from $25000 \mathrm{ng} / \mathrm{mL}$ DBS samples. In the absence of a significant 
TABLE 3: Stability data for caffeine in DBS samples collected on FTA Elute paper stored at room temperature with desiccant for 6 days $n=6$.

\begin{tabular}{lcccc}
\hline \multirow{2}{*}{ Nominal conc. (ng/mL) } & \multicolumn{2}{c}{1000} & \multicolumn{2}{c}{20000} \\
& Fresh & Stored & Fresh & Stored \\
\hline Mean conc. (ng/mL) & 955 & 1034 & 18377 & 20054 \\
SD & 99 & 95 & 819 & 1057 \\
CV\% & 10.3 & 9.2 & 4.5 & 5.3 \\
\% Difference & \multicolumn{2}{c}{+8.3} & \multicolumn{2}{c}{+9.1} \\
\hline
\end{tabular}

peak attributable to caffeine in resulting chromatograms the punching device was also excluded as a major factor affecting assay accuracy and precision.

\section{Conclusions}

The accuracy (RE\%) and precision (CV\%) results for the developed LC-MS method were within the accepted validation criteria of less than $20 \%$ at the LLOQ and less than $15 \%$ at all other tested concentrations. A linear response was observed up to a DBS concentration of $25000 \mathrm{ng} / \mathrm{mL}$ with a mean $r^{2}=0.9960$. Reports by Lee et al. [20, 21] suggest that during the treatment of AoP, the lowest anticipated caffeine concentration is $\sim 2500 \mathrm{ng} / \mathrm{mL}$ and therefore an LLOQ of $500 \mathrm{ng} / \mathrm{mL}$ is more than adequate.

There were no significant interferences at the retention time of caffeine and IS in any of the control DBS chromatograms or in samples collected from two adult volunteers demonstrating the selectivity of the developed analytical techniques. The results obtained here indicate the potential of LC-MS (SIM) to provide a selective method for the quantification of caffeine within DBS samples under the sample preparation conditions used. Results from the matrix effect experiment suggested significant ion suppression from the FTA Elute paper itself and demonstrate the importance of understanding the source and extent of the matrix effects.

\section{Acknowledgments}

The authors acknowledge the help of Dr. Hitesh Pandya, University of Leicester, for guidance with the clinical aspects of the study.

\section{References}

[1] R. Guthrie and A. Susi, "A simple phenylalanine method for detecting phenylketonuria in large populations of newborn infants," Pediatrics, vol. 32, pp. 338-343, 1963.

[2] S. Tanna and G. Lawson, "Analytical methods used in conjunction with dried blood spots," Analytical Methods, vol. 3, no. 8, pp. 1709-1718, 2011.

[3] P. M. Edelbroek, J. V. D. Heijden, and L. M. L. Stolk, "Dried blood spot methods in therapeutic drug monitoring: methods, assays, and pitfalls," Therapeutic Drug Monitoring, vol. 31, no. 3, pp. 327-336, 2009.

[4] W. Li and F. L. S. Tse, "Dried blood spot sampling in combination with LC-MS/MS for quantitative analysis of small molecules," Biomedical Chromatography, vol. 24, no. 1, pp. 4965, 2010.

[5] P. Patel, S. Tanna, H. Mulla, V. Kairamkonda, H. Pandya, and G. Lawson, "Dexamethasone quantification in dried blood spot samples using LC-MS: the potential for application to neonatal pharmacokinetic studies," Journal of Chromatography B, vol. 878, no. 31, pp. 3277-3282, 2010.

[6] G. Lawson, E. Cocks, and S. Tanna, "Quantitative determination of atenolol in dried blood spot samples by LC-HRMS: as potential method for assessing medication adherence," Journal of Chromatography B, vol. 897, pp. 72-79, 2012.

[7] G. Lawson, H. Mulla, and S. Tanna, "Captopril determination in dried blood spot samples with LC-MS and LC-HRMS: a potential method for neonate pharmacokinetic studies," Journal of Bioanalysis and Biomedicine, vol. 4, no. 2, pp. 16$25,2012$.

[8] J. V. Mei, J. R. Alexander, B. W. Adam, and W. H. Hannon, "Use of filter paper for the collection and analysis of human whole blood specimens," Journal of Nutrition, vol. 131, no. 5, pp. 1631S-1636S, 2001.

[9] N. Spooner, R. Lad, and M. Barfield, "Dried blood spots as a sample collection technique for the determination of pharmacokinetics in clinical studies: considerations for the validation of a quantitative bioanalytical method," Analytical Chemistry, vol. 81, no. 4, pp. 1557-1563, 2009.

[10] M. Barfield, N. Spooner, R. Lad, S. Parry, and S. Fowles, "Application of dried blood spots combined with HPLC-MS/ MS for the quantification of acetaminophen in toxicokinetic studies," Journal of Chromatography B, vol. 870, no. 1, pp. 3237, 2008.

[11] J. L. Brazier, D. Delaye, M. Desage, and A. Bannier, "Simultaneous microdetermination of theophylline, caffeine and phenobarbital in blood collected on paper," Journal of Chromatography, vol. 224, no. 3, pp. 439-448, 1981.

[12] R. Lad, "Validation of individual quantitative methods for determination of cytochrome $\mathrm{P} 450$ probe substrates in human dried blood spots with HPLC-MS/MS," Bioanalysis, vol. 2, no. 11, pp. 1849-1861, 2010.

[13] "ICH Harmonised tripartite guideline note for guidance on toxicokinetics: the assessment of systemic exposure in toxicity studies S3A," 1994, http://www.ich.org/fileadmin/Public_ Web_Site/ICH_Products/Guidelines/Safety/S3A/Step4/S3A Guideline.pdf.

[14] Guidance For Industry Bioanalytical Method Validation, Food and Drug Administration, Rockville, Md, USA, 2001.

[15] X. Guo and E. Lankmayr, "Phospholipid-based matrix effects in LC-MS bioanalysis," Bioanalysis, vol. 3, no. 4, pp. 349-352, 2011.

[16] M. Lahaie, J. N. Mess, M. Furtado, and F. Garofolo, "Elimination of LC-MS/MS matrix effect due to phospholipids using specific solid-phase extraction elution conditions," Bioanalysis, vol. 2, no. 6, pp. 1011-1021, 2010.

[17] G. T. Clark, J. J. Haynes, M. A. J. Bayliss, and L. Burrows, "Utilization of DBS within drug discovery: development of a serial microsampling pharmacokinetic study in mice," Bioanalysis, vol. 2, no. 8, pp. 1477-1488, 2010.

[18] G. Liu, L. Patrone, H. M. Snapp et al., "Evaluating and defining sample preparation procedures for DBS LC-MS/MS assays," Bioanalysis, vol. 2, no. 8, pp. 1405-1414, 2010.

[19] A. van Eeckhaut, K. Lanckmans, S. Sarre, I. Smolders, and Y. Michotte, "Validation of bioanalytical LC-MS/MS assays: evaluation of matrix effects," Journal of Chromatography B, vol. 877 , no. 23, pp. 2198-2207, 2009. 
[20] T. C. Lee, B. Charles, P. Steer, V. Flenady, and A. Shearman, "Population pharmacokinetics of intravenous caffeine in neonates with apnea of prematurity," Clinical Pharmacology and Therapeutics, vol. 61, no. 6, pp. 628-640, 1997.

[21] H. S. Lee, Y. M. Khoo, Y. Chirino-Barcelo, K. L. Tan, and D. Ong, "Caffeine in apnoeic Asian neonates: a sparse data analysis," British Journal of Clinical Pharmacology, vol. 54, no. 1, pp. 31-37, 2002. 


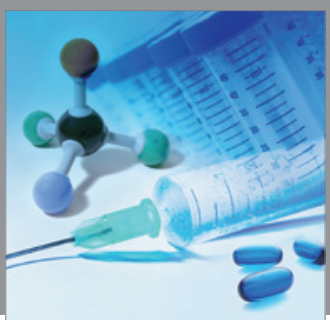

International Journal of

Medicinal Chemistry

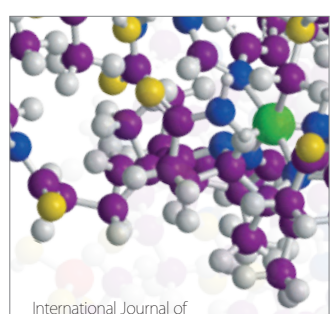

Carbohydrate Chemistry

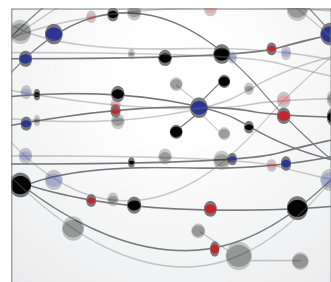

The Scientific World Journal
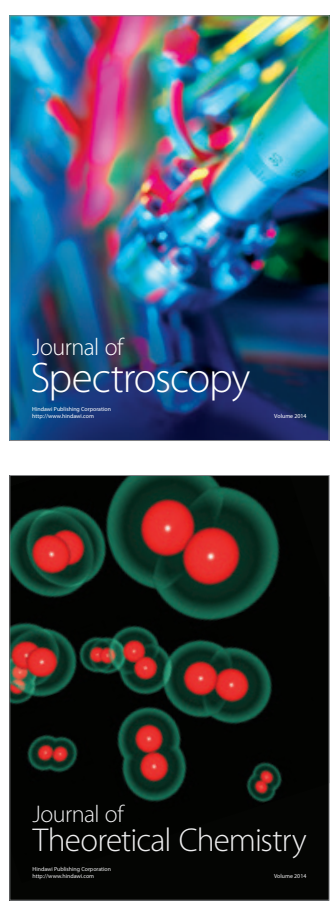
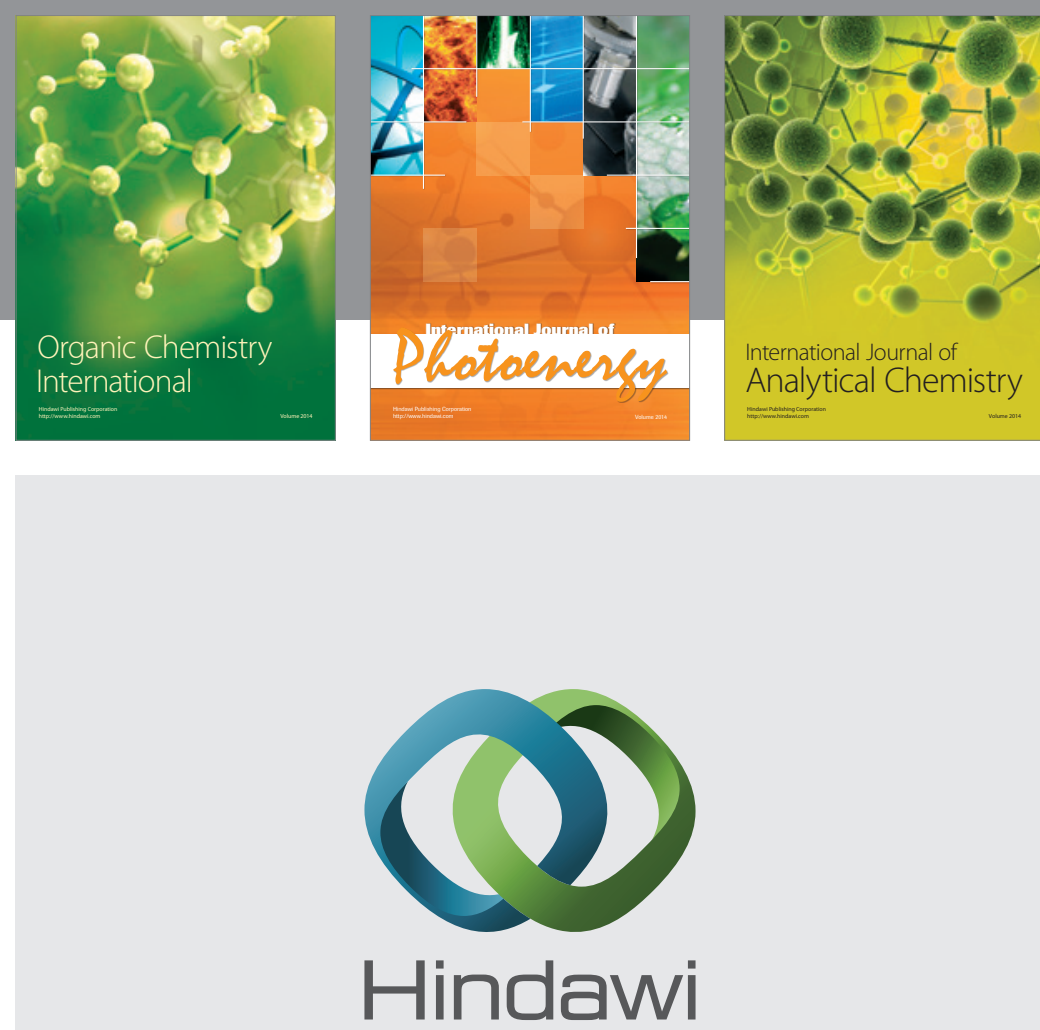

Submit your manuscripts at

http://www.hindawi.com
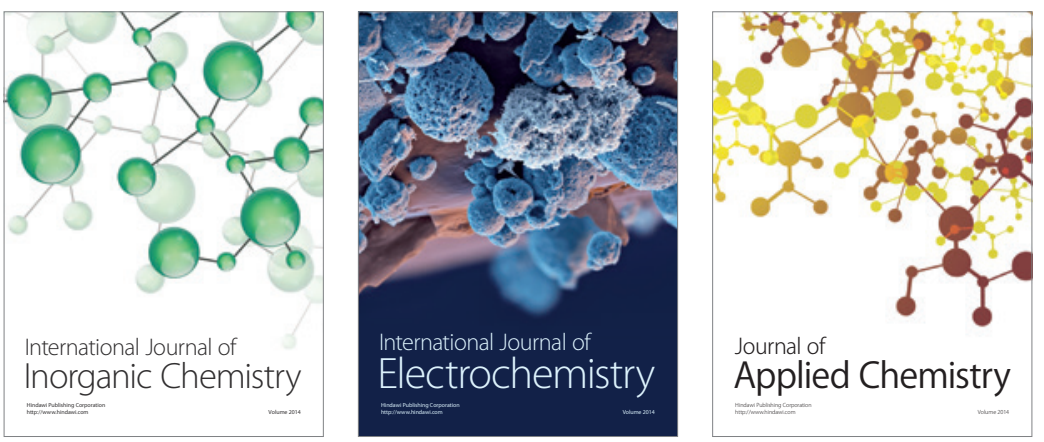

Journal of

Applied Chemistry
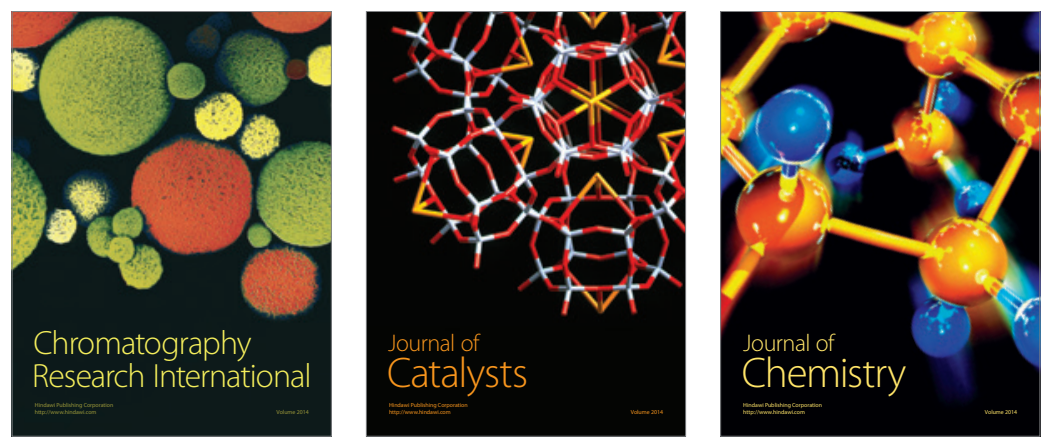
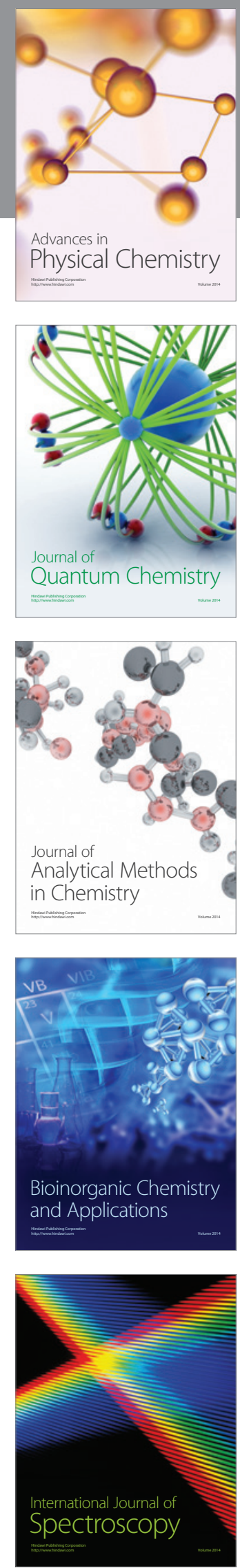